\title{
SIGNAL NOISE REDUCTION BASED ON WAVELET TRANSFORM IN TWO-WAVELENGTH LIDAR SYSTEM
}

\author{
Shuo Shi ${ }^{\text {a, } * \text {, Wei Gong }}{ }^{\text {a }}$, Lilei Lv ${ }^{\mathrm{b}}$, Bo Zhu ${ }^{\text {a }}$, Shalei Song ${ }^{\mathrm{c}}$ \\ ${ }^{a}$ State Key Laboratory of Information Engineering in Surveying, Mapping and Remote Sensing, Wuhan University, \\ Wuhan, Hubei 430079, China \\ ${ }^{\mathrm{b}}$ China Petroleum Engineering Limited liability company in North China branch (CPENC), Jianshe Road, Renqiu, \\ Hebei 062552, China \\ ${ }^{\mathrm{c}}$ Wuhan Institute of Physics and Mathematics, Chinese Academy of Sciences, Wuhan, Hubei 430071, China
}

Commission VII/7

KEY WORDS: LIDAR, Data mining, Proceedings, Algorithms, Estimation

\begin{abstract}
:
Nowadays lidar has become a leading edge technology for photogrammetry and remote sensing. A new lidar with two detection wavelengths was presented in this paper. The two-wavelength lidar can obtain spatial information and spectral information, and intensity of backscatter signals in two-wavelength can be availably used for objects identification and observation. However, the backscatter signals are weak and strongly influenced by the noise. In order to acquire accurate intensity, the signal noise has to be removed. As a useful tool for signal processing, wavelet transform was chosen. In this study, the principle of two-wavelength lidar was introduced and possible noise in the backscatter signal was analysed. Then the signal progressing was executed on the basic of wavelet de-noising. First, singular values caused by varieties of noise source have to be rejected from the signal based on three standard deviations. Second, combination of modulus maxima method and threshold method is used to make further improvement on the signal. Finally, an evaluation of the noise reduction was given to testify the ability of the method. The results show that the effect of the method based on wavelet transform is better than other classical algorithms, and the method is suitable for signal noise reduction in two-wavelength lidar system.
\end{abstract}

\section{INTRODUCTION}

As an active measurement way of remote sensing, airborne laser scanning (ALS) has become a well-established technology for the detection of topography and for mapping the Earth's surface (A. Wehr and U. Lohr, 1999; K. Kraus and N. Pfeifer, 1998). However, the detection information of target characteristics by traditional lidar is poor because of the single-wavelength measurement ( $\mathrm{J}$ Grace, et al, 2007). A new lidar with two detection wavelengths was provided by Wuhan University to improve the obtained information about properties of surface objects. Not only spatial information of objects but also spectral information can be acquired by the two-wavelength lidar, and intensity of backscatter signals in two-wavelength can be availably used for objects identification and observation. Furthermore, the two-wavelength lidar might be widely used for monitoring in the growth and nutrition status of vegetation.

The two-wavelength lidar has been successfully developed and was used for features monitoring in the laboratory environment. As is well known, the backscatter signals of lidar are very weak. And it is difficult to obtain high-quality signals, especially in the condition of low-power transmitters and strong absorption on the objects. Besides, the weak signals received might be strongly influenced by all kinds of noise from the equipment and the ambient environment. In fact, the final signals acquired by the instrument are so poor that further application is hard to continue. As a result, the signal noise has to be removed in order to get accurate information.

Signal noise reduction based on wavelet transform has become the hot spot of academia right now. It is an effective method to reduce the noise in signal by wavelet multiscale decomposition (Liang Zhang, et al, 2002), and was applied for de-noising of backscatter signals in two-wavelength lidar system. In this paper, system description of two-wavelength lidar was given and the backscatter signals were presented. After the analysis of characteristic of noise, combination method of modulus maxima method and threshold method was proposed, and it was testified to be an effective algorithm to reduce the signal noise.

\section{SYSTEM DESCRIPTION AND NOISE ANALYSIS}

\subsection{System Description}

The new breadboard instrument of two-wavelength lidar system has been developed. The system is mainly composed of three parts: the transmitting sub-system, the optical receiver subsystem and data acquisition and processing sub-system. The block diagram of the two-wavelength lidar system is shown in Figure 1.

\footnotetext{
* Corresponding author.

E-mail address: shishuo@whu.edu.cn
} 


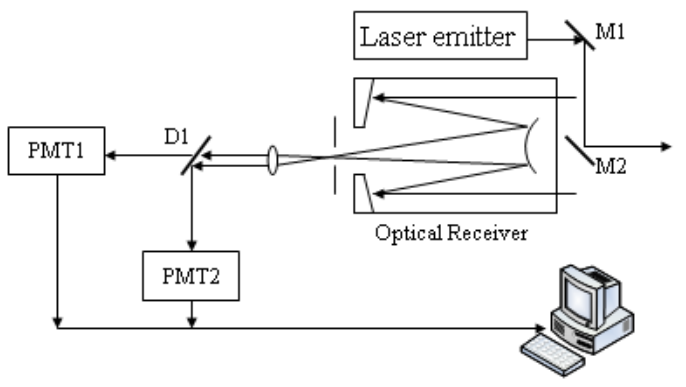

Figure 1. The block diagram of two-wavelength lidar

The two-wavelength lidar system works as follows. The laser emitter transmits the laser bean separately in red and nearinfrared range. The laser light will be reflected to detect objects through the holophote composed of mirrors M1 and M2. Then backscatter signals can be received by the Schmidt-Cassegrain telescope with $200 \mathrm{~mm}$ diameters, and be divided into two wavelengths through dichroic filters D1. Subsequently, PMT1 and PMT2 are used to transform optical to electrical as the devices of photo-electric detection. Finally data acquisition and processing sub-system in the computer can obtain the backscatter intensities of the objects.

\subsection{Noise analysis}

After photo-electric detection, backscatter signals can be acquired by the data acquisition sub-system of two-wavelength lidar. There are two different signals respectively in red channel and near infrared channel as shown in Figure 2.

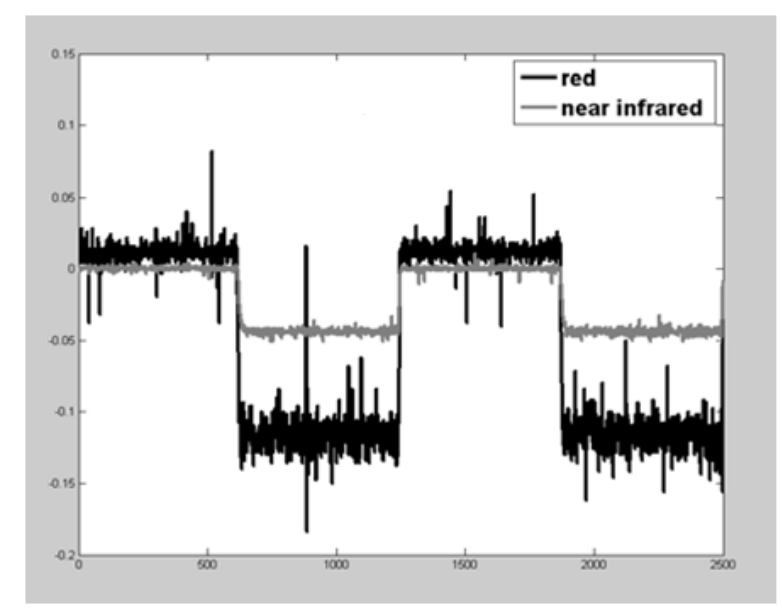

Figure 2. Backscatter signals of two-wavelength lidar

It can be seen from the Figure 2 that the backscatter signals received by the system are so bad that we cannot use directly for further calculation. The possible noise and sources should be analyzed before removal of noise.

Dark current, background radiation noise and circuit noise caused by the detector and external environmental factors can seriously affect the backscatter signals. The noise from the sources is unrelated to each other in statistics. In the limited bandwidth of the signal, the noise could be regarded as white noise approximately and subject to normal distribution. Besides, there will also be some colored noise due to the remaining multimode interference effect of the laser beam. However, the generated thermal noise and shot noise can be neglected compared with dark current, background radiation noise and circuit noise. Thus, noise of backscatter signals in twowavelength lidar system can be removed as the Gaussian white noise

\section{METHOD BASED ON WAVELET TRANSFORM}

\subsection{Principle of wavelet transform}

Wavelet transform could map the signal to a group of basic functions which were generated by the wavelet expansion and shifting from. Thus the reasonable separation of signal could be made both in different frequency bands and different moments in the fact. Wavelet transform provides an effective tool for nonstationary description of the dynamic signals and the extraction of weak signal.

\subsection{De-noising process}

Traditionally, the de-nosing process based on wavelet transform mainly consists of three steps: firstly, wavelet decomposition of the leakage signals, for which we must select the best mother wavelet and scale; secondly, threshold quantization of decomposing coefficients was made; finally, reconstruction wavelet de-noising signals was carried out.

Especially, based on the characteristics of the backscatter signals, some of de-noising process in the two-wavelength lidar system should be discussed in detail.

\subsubsection{Wavelet decomposition}

The wavelet transform is used to perform a multiscale decomposition on the noisy signals. More specifically, the corresponding wavelet coefficients are got by choosing suitable wavelet and wavelet decomposition layers. Daubechies wavelet function can effectively avoid the signal phase shifter and can also achieve better smoothing effect in the process of reconstruction on the basic of its symmetry and regularity property. As a result, three orders Daubeachies wavelet was chosen as the basic wavelet function in the de-noising process. Besides, the noisy signal was decomposed to 4 layers.

\subsubsection{Removal of the singular value}

Through the analysis of characteristics of the backscatter signals, there are many singular values caused by varieties of noise sources in the signals. What's worse, the de-nosing result will be seriously affected if the singular values can not be removed.

It is very convenient to distinguish the parts of noise and saltation of signal by $3 \sigma$-rule applied in electronic measurement (HE Shi-biao, et al, 2002). The probability of the absolute value greater than three standard deviations is only about $3 \%$, and all the values which are greater than $3 \sigma$ can be regarded as the coarse errors. In this way, singular values could be removed effectively.

\subsubsection{Threshold quantification}

In order to extract the wavelet coefficients of signals and remove the wavelet coefficients of noise in the scales, a threshold should be chosen to carry out the quantification from the first level to the $\mathrm{N}$ layer of high frequency coefficients. In the real applications, there are many methods to solve the problem. 
An algorithm combining threshold method and modulus maxima method was chosen in the de-nosing process. Through the theory of tracking maximum modulus in the scales, modulus maxima method can search the characteristic points of the signals in the lower scale on the basic of the maximum modulus chosen in the highest scale (MALLAT S, 1992). However, a lot of noise is contained in the detail information of the first scale. Thus, noise cannot be completely reduced because the reserved modulus maximum points often contain part of noise. To solve the problem, threshold method was applied to combine with the modulus maxima method. In detail, principle of wavelet entropy was introduced in the first scale to obtain the adaptive threshold value (Zhengyou He, et al, 2004).

\section{EXPERIMENT AND EVALUATION}

\subsection{Experiment}

The de-nosing method based on the combination of threshold method and modulus maxima method was proposed and experiment should be carried out to testify the ability of reducing signal noise. In the two-wavelength lidar system, there are two backscatter signals respectively in red channel and near infrared channel. In order to better display the ability of denosing, the worse signal which is in red channel was chosen.

According to the proposed method based on wavelet transform, the de-noising process was made according to the flow shown in the Figure 3.

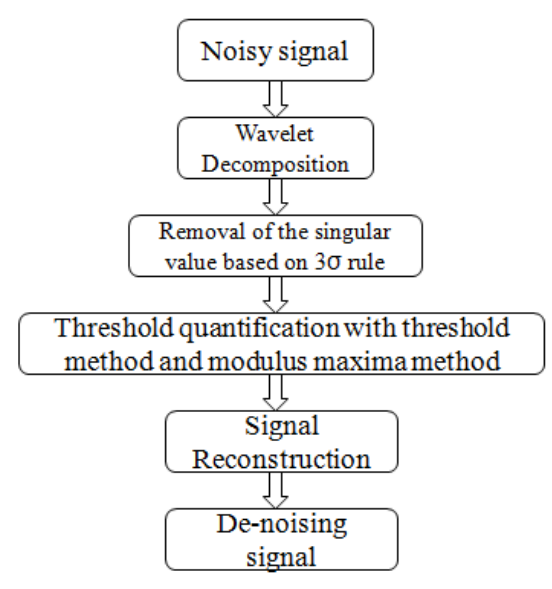

Figure. 3 Flow of de-noising process

"db3" was chosen to be the basis function of wavelet transform, and the noisy signal was decomposed to 4 layers. Firstly, the mean square value of the high-frequency coefficients was calculated to remove the singular values, and all the wavelet transform coefficients greater than $3 \sigma$ were set to zero while the others remain unchanged. Then further improvement on the signal is made based on the combination of modulus maxima method and threshold method. Finally the decomposition coefficients were reconstructed by remodelling function, and the result of the reconstruction was de-noising signal.

\subsection{De-noising effect evaluation}

In order to testify the ability of the method, an evaluation of the noise reduction should be given. As a result, some other classical algorithms were applied in the experiment to make comparison with the combination method of modulus maxima method and threshold method. Direct average method, forced de-noising method and FFT method were chosen, and the result of de-noising is shown in the Figure 4.

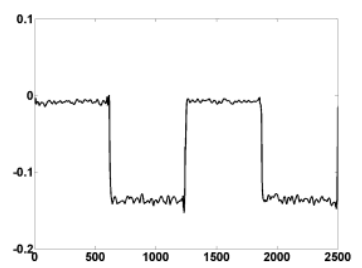

a. Combination method

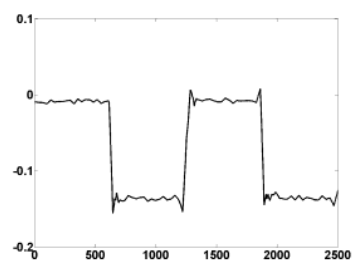

c. Forced de-noising method

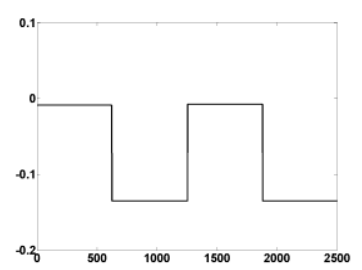

b. Direct average method

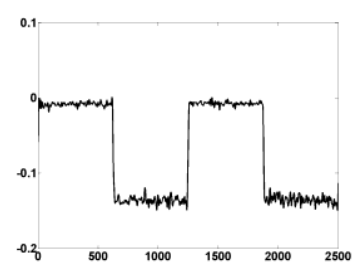

d. FFT method
Figure 4. Comparison of different de-noising algorithms

As the Figure 4 shows, the backscatter signal becomes smooth after de-noising of all the algorithms. However, the effects of different algorithms are obviously different. The noisy signal reduced by combination method has most smooth waveform and has best effeteness. On the contrary, direct average method makes the signal lose some useful component, while there were still peaks exist in the edge of the signal by forced de-noising method. For the FFT method, noise cannot be correctly reduced from the signal because of the lack of local analysis.

Besides, measurement criteria were also defined to further evaluate the effects of signal noise reduced. In detail, signal-tonoise ratio (SNR) and mean-square deviation (RMSE) are expressed as the measurement criteria in the following calculation formulas.

$$
S N R=10 \times \log _{10}(S / N)
$$

Where $\quad S=$ power of raw signal

$\mathrm{N}=$ power of noise

$$
R M S E=\sqrt{\frac{1}{n} \sum_{n}(\hat{f(n)}-f(n))^{2}}
$$

Where

$$
\begin{aligned}
& \hat{f(n)}=\text { de-noising signal } \\
& f(n)=\text { raw signal }
\end{aligned}
$$

The effects of de-noising will be better when SNR are higher and RMSE are smaller. SNR and RMSE of different algorithms were calculated and the results were shown in the Table 1. 


\begin{tabular}{|c|c|c|}
\hline Algorithm & SNR & RMSE \\
\hline Combination method & 21.94 & 0.0065 \\
Direct average method & 17.54 & 0.0110 \\
Forced de-noising method & 19.00 & 0.0093 \\
FFT method & 21.34 & 0.0070 \\
\hline
\end{tabular}

Table 1. SNR and RMSE of different de-noising algorithms

In the Table 1, the SNR of combination method is highest and the RMSE is smallest. So, the combination of modulus maxima method and threshold method based on wavelet transform can effectively eliminate different noise of backscatter signals and make the extracted signal cognizable.

\section{CONCLUSIONS}

In this paper, a new lidar with red and near infrared wavelengths was proposed to apply for features monitoring of different objects. However, backscatter signals of the lidar system are seriously strongly influenced by variety of noise and signal noise which has to be reduced. Based on the wavelet transform, combination method of modulus maxima method and threshold method was proposed. The results tested the ability of the algorithm after comparison of other classical algorithms. In fact, the de-noised signals had smooth waveforms and the SNR was also improved significantly after the de-noising processing. Nevertheless, there are still some questions over this method, and the algorithm should be further improved in the subsequent work.

\section{REFERENCES}

A. Wehr and U. Lohr, 1999. Airborne laser scanning-An introduction and overview. ISPRS Journal of Photogrammetry and Remote Sensing, 54(2/3), pp. 68-82.

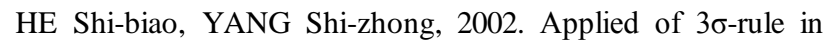
Reducing Noise in Signal by Wavelet analysis. Journal of Chongqing University(Natural Science Edition), 25(12), pp.5861 .

J Grace, C Nichol, et al, 2007. Can we measure terrestrial photosynthesis from space directly, using spectral reflectance and fluorescence. Global Change Biology, 13(7), pp.1484-1497.

K. Kraus and N. Pfeifer, 1998. Determination of terrain models in wooded areas with airborne laser scanner data. ISPRS Journal of Photogrammetry and Remote Sensing, 53(4), pp. 193-203.

Liang Zhang, Demin Wang, et al, 2002 . Adaptive SPIHT for image coding based on cured wavelet transform. The International Society for Optical Engineering, 5685, pp. 160163.

MALLAT S. Singularity detection and processing with wavelets. IEEE Transactions on Information Theory, 1992. 38(2), pp.617 $-643$.

Zhengyou He, Yumei Cai, et al, 2004. A study of wavelet entropy theory and its application in power system. IEEE
International Conference on Intelligent Mechatronics and Automation, 8, pp.847-851.

\section{ACKNOWLEDGEMENTS}

This work was supported by 973 Program (2009CB723905), the NSFC (41101334, 10978003, 41127901), the Fundamental Research Funds for the Central Universities. 